\section{Management of glaucoma in patients with nanophthalmos}

\begin{abstract}
Aims In this retrospective study, we aimed to evaluate the results of glaucoma surgery in patients with nanophthalmos.

Methods Twenty-eight bilateral

nanophthalmic patients, of whom 20 patients underwent trabeculectomy + Mitomycin-C (MMC) + inferior sclerotomy between 1996 and 2004, were included in this study. Records of patients with nanophthalmos were reviewed. Intraocular pressure (IOP), glaucoma medications, surgical success, visual acuity and complications were analyzed.

Results The mean IOP was $34.6 \pm 5.3 \mathrm{~mm} \mathrm{Hg}$ preoperatively. At the final follow-up visit, the mean IOP dropped to $21.41 \pm 7.34 \mathrm{~mm} \mathrm{Hg}$ $(P<0.05)$. The cumulative probability of success was $85 \%$ at 1 year, $78.5 \%$ at 2 years, $76.9 \%$ at 3 years, $70.6 \%$ at 4 years and $47 \%$ at 5 years after surgery. Visual acuity decreased in $13(65 \%)$ patients but no eye lost vision.

Sequels of choroidal detachment (five patients $25 \%$ ) and retinal folds (four patients $20 \%$ ) were the most frequent reason for visual decrease. Uveal effusion (10 patients $50 \%$ ) and cataract formation (seven patients $35 \%$ ) were major late postoperative complications.

Conclusions Results indicate that trabeculectomy + MMC + inferior sclerotomy procedure was effective and safe for glaucoma control in patients with nanophthalmos, but uveal effusion is a major problem and cataract surgery is expected in the long run.

Eye (2008) 22, 838-843; doi:10.1038/sj.eye.6702742; published online 9 February 2007
\end{abstract}

The authors have no proprietary interest in any of the materials or techniques used in this study. Presented in part as a free paper presentation at the Annual Meeting of the American Academy of Ophthalmology, Chicago, IL; October 2005
Keywords: nanophthalmos; glaucoma; microphthalmos; trabeculectomy; Mitomycin-C; inferior sclerotomy
IS Yalvac ${ }^{1,2}$, B Satana1', G Ozkan¹, U Eksioglu and S Duman ${ }^{1}$

\section{Introduction}

Nanophthalmos is a relatively rare abnormality characterized by a small eye without other malformations. Clinical features that have been described include a narrow palpebral fissure, a deeply set globe in a small orbit, a short axial length, an abnormally thickened sclera, axial hypermetropia, small to normal corneal diameter, a shallow anterior chamber and high lens/eye volume ratio. ${ }^{1,2}$ Management of glaucoma in these patients is challenging. Glaucoma filtration surgery is considered as the last choice because of the high incidence of intraoperative and postoperative complications. ${ }^{3,4}$

The purpose of this study is to evaluate the effect and safety of filtrating glaucoma surgery in patients with nanophthalmos.

Patients and methods

\section{Patients}

We evaluated the medical records of all patients with diagnosis of nanophthalmos at the Glaucoma Department of Ankara Research and Education Hospital. Nanophthalmos was distinguished from other types of microphthalmia and relative anterior microphthalmos (RAM) ${ }^{5}$ and was diagnosed based on a shorter than average axial length $(<20.5 \mathrm{~mm})$, a shallow anterior chamber, high lens/eye volume ratio, moderate to severe hyperopia, and diffused choroidal-scleral thickening as shown by echography. Among the 32 records, 28 patients were identified as having bilateral nanophthalmos. Patients who did not undergo glaucoma surgery or inadequate records to ensure at least 1 year of follow-up were excluded from the study. The records of 20 eyes of 20 patients with bilateral nanophthalmos who underwent trabeculectomy + Mitomycin-C 
$(\mathrm{MMC})+$ inferior sclerotomy were retrospectively analyzed between October 1996 and January 2004. All the surgeries were done by one surgeon (ISY). In seven patients who underwent bilateral surgery, only the first eye was included in the study. Ethics Committee for Human Research at the Ankara Research and Education Hospital approved this study. All of the 20 eyes with angle-closure glaucoma were treated with laser iridotomy as the initial procedure with simultaneous argon laser peripheral iridoplasty (ALPI) in two eyes.

The patient charts were reviewed for sex, age at initial diagnosis, family history, visual acuity, refraction, corneal diameter, keratometry, gonioscopy, intraocular pressure (IOP), echographic findings (anterior chamber depth, lens and scleral thickness, axial length), fundus examination, glaucoma medications and postoperative complications.

\section{Surgical technique}

Before surgery, an intravenous hyperosmotic agent was given to all patients to shrink vitreous and minimize posterior pressure. The trabeculectomies were performed using a limbus-based conjunctival flap and modified Cairns technique at the superior quadrant. Intraoperative MMC (Mitomycin-C ${ }^{\circledR}$, Kyowa Hakko Kogyo Co. Ltd, Tokyo, Japan) in a concentration of $0.2 \mathrm{mg} / \mathrm{ml}$ was applied using a surgical sponge for $2 \mathrm{~min}$. Major modifications of our trabeculectomy procedures were preplaced scleral flap sutures, tight suture closure, leaving viscoelastic material in the anterior chamber and performing inferior sclerotomies at two sites in temporoinferior and nasoinferior quadrants. The margin of the sclerotomy was located about $3 \mathrm{~mm}$ behind the limbus anteriorly. The edges of the scleral wound were cauterized by diathermy to prevent adhesion resulting from scarring. The sclerotomies were not sutured but Tenon's capsule and the bulbar conjunctiva were closely sutured. We did not perform decompression of the vortex vein or sclerotomy. Subconjunctival or subtenon injection of $10 \mathrm{mg} / \mathrm{ml}$ triamcinolone acetonide (Kenacort ${ }^{\circledR}-A$, Bristol Myers Squibb Co., Princeton, NJ, USA) was used preoperatively to assist in postoperative inflammation and uveal effusion control.

All the patients received prednisolone acetate $1 \%$ (Pred Forte ${ }^{\circledR}$ Allergan, Irvine, CA, USA) topically for every $2 \mathrm{~h}$, starting from the day after surgery. After 1 week, the administration of the corticosteroids was decreased to four times a day, tapering gradually over 8-week period. Cyclopentolate 1\% drops were used for a month postoperatively. Oral prednisolone was used as 1$1.5 \mathrm{mg} / \mathrm{kg}$, which gradually tapered over 8-week period. Since trabeculectomy flap is tied strongly, laser suture lysis was performed being initiated 1 week after surgery in six $(30 \%)$ patients.

\section{Criteria for success and failure}

Criteria for success were defined as IOP $<22 \mathrm{~mm} \mathrm{Hg}$ and $>5 \mathrm{~mm} \mathrm{Hg}$ without a further glaucoma surgery performed and loss of light perception. Postoperative use of antiglaucoma medications was not a criterion for success or failure.

\section{Statistical analysis}

Results are expressed as mean \pm standard deviation (SD). With the SPSS (SPSS for Windows, version 12.0, SPSS, Chicago, IL, USA) software, analyses of continuously scaled variables before and after surgery were made using the paired $t$-test and Wilcoxon-signed rank test. The cumulative probability of success was analyzed by Kaplan-Meier life-table analysis. $P$-values $<0.05$ were considered statistically significant.

\section{Results}

\section{Patient characteristics}

Among the 28 patients with diagnosis of nanophthalmos, 20 of them $(71.4 \%)$ underwent

trabeculectomy $+\mathrm{MMC}+$ inferior sclerotomy, with eight (40\%) patients being female and $12(60 \%)$ patients being male. The mean follow-up at the initial diagnosis at our department was 55.70 \pm 34.88 months (range 55-124 months). The mean follow-up after glaucoma surgery was $41.20 \pm 6.88$ months (range 12-109 months). Mean age was $33.06 \pm 15.26$ years (range 15-67 years) at the initial diagnosis. A history of consanguinity among the parents was present in $12(60 \%)$ out of the 20 patients. A family history of nanophthalmos was reported in 10 $(50 \%)$ patients. All patients had revealed closed angles Grade 1 or 0 with gonioscopy. There was variable hyperopia of +5 to +15 diopters (D) spherical equivalent (mean $10.75 \pm 2.69 \mathrm{D}$ ). The mean corneal diameter was $10.54 \pm 0.89 \mathrm{~mm}$ (range $8-11.5 \mathrm{~mm}$ ). The mean axial length was $17.59 \pm 1.65 \mathrm{~mm}$ (range 14.91-20.34 mm). The mean anterior chamber depth was $2.30 \pm 0.36 \mathrm{~mm}$ (range $1.80-2.98 \mathrm{~mm}$ ). The mean lens thickness was $5.00 \pm 0.89 \mathrm{~mm}$ (range $3.09-6.93 \mathrm{~mm}$ )

(Table 1).

\section{Intraocular pressure}

Mean IOP was $34.6 \pm 5.34 \mathrm{~mm} \mathrm{Hg}$ (range $27-54 \mathrm{~mm} \mathrm{Hg}$ ) preoperatively. Mean IOPs were $14.40 \pm 5.02 \mathrm{~mm} \mathrm{Hg}$ (range $10-27 \mathrm{~mm} \mathrm{Hg}$ ) at 1 year, $15.85 \pm 4.95 \mathrm{~mm} \mathrm{Hg}$ 
Table 1 Demographic data

\begin{tabular}{|c|c|}
\hline \multicolumn{2}{|c|}{ Follow-up at initial diagnosis (months) } \\
\hline Mean \pm SD & $55.70 \pm 34.88$ \\
\hline Range & $55-124$ \\
\hline \multicolumn{2}{|l|}{ Age (years) } \\
\hline Mean \pm SD & $33.06 \pm 15.26$ \\
\hline Range & $15-67$ \\
\hline \multicolumn{2}{|l|}{ Gender } \\
\hline Female & $8(40 \%)$ \\
\hline Male & $12(60 \%)$ \\
\hline \multicolumn{2}{|c|}{ Follow-up after surgery (months) } \\
\hline Mean \pm SD & $41.20 \pm 6.88$ \\
\hline Range & $12-109$ \\
\hline \multicolumn{2}{|c|}{ Preoperative medications } \\
\hline Mean \pm SD & $2.30 \pm 0.88$ \\
\hline Range & $2-3$ \\
\hline \multicolumn{2}{|c|}{ Postoperative medications } \\
\hline Mean \pm SD & $1.60 \pm 2.23$ \\
\hline Range & $0-3$ \\
\hline \multicolumn{2}{|c|}{ Horizontal corneal diameter ( $\mathrm{mm}$ ) } \\
\hline Mean \pm SD & $10.54 \pm 0.89$ \\
\hline Range & 8-11.5 \\
\hline \multicolumn{2}{|l|}{ Axial length (mm) } \\
\hline Mean \pm SD & $17.59 \pm 1.65$ \\
\hline Range & $14.91-20.34$ \\
\hline \multicolumn{2}{|c|}{ Anterior chamber depth ( $\mathrm{mm})$} \\
\hline Mean \pm SD & $2.30 \pm 0.36$ \\
\hline Range & $1.80-2.98$ \\
\hline \multicolumn{2}{|c|}{ Lens thickness $(\mathrm{mm})$} \\
\hline Mean \pm SD & $5.00 \pm 0.89$ \\
\hline Range & $3.09-6.93$ \\
\hline \multicolumn{2}{|l|}{ Refraction (D) } \\
\hline Mean $\pm S D$ & $10.75 \pm 2.69$ \\
\hline Range & 5-15 \\
\hline \multicolumn{2}{|l|}{ Keratometry $(D)$} \\
\hline Mean \pm SD & $48.27 \pm 2.29$ \\
\hline Range & $45.00-55.00$ \\
\hline Family history & $10(50 \%)$ \\
\hline Consaguinity & $12(60 \%)$ \\
\hline Total & $20(100 \%)$ \\
\hline
\end{tabular}

Abbreviations: D, diopters; SD, standard deviation.

(range $11-29 \mathrm{~mm} \mathrm{Hg}$ ) at 2 years, $18.34 \pm 6.23 \mathrm{~mm} \mathrm{Hg}$ (range $12-34 \mathrm{~mm} \mathrm{Hg}$ ) at 3 years, $17.50 \pm 7.28 \mathrm{~mm} \mathrm{Hg}$ (range $14-31 \mathrm{~mm} \mathrm{Hg}$ ) at 4 years and $18.34 \pm 6.23 \mathrm{~mm} \mathrm{Hg}$ (range 12-34 $\mathrm{mm} \mathrm{Hg}$ ) at 5 years postoperatively. Pre- and postoperative IOP differences were statistically significant at all periods respectively $(P<0.001)$. The
Table 2 Mean IOP profile before and after surgery in patients with nanophthalmos

\begin{tabular}{lcc}
\hline & $I O P \pm S D(\mathrm{n}=20)$ & $P^{\mathrm{a}, \mathrm{b}}$ \\
\hline Preoperative & $34.6 \pm 5.34 \mathrm{~mm} \mathrm{Hg}(27-54 \mathrm{~mm} \mathrm{Hg})$ & \\
& & \\
Postoperative & & \\
1 year & $14.40 \pm 5.02 \mathrm{~mm} \mathrm{Hg}(10-27 \mathrm{~mm} \mathrm{Hg})$ & $<0.001$ \\
2 years & $15.85 \pm 4.95 \mathrm{~mm} \mathrm{Hg}(11-29 \mathrm{~mm} \mathrm{Hg})$ & $<0.001$ \\
3 years & $18.34 \pm 6.23 \mathrm{~mm} \mathrm{Hg}(12-34 \mathrm{~mm} \mathrm{Hg})$ & $<0.001$ \\
4 years & $17.50 \pm 7.28 \mathrm{~mm} \mathrm{Hg}(14-31 \mathrm{~mm} \mathrm{Hg})$ & $<0.001$ \\
5 years & $18.34 \pm 6.23 \mathrm{~mm} \mathrm{Hg}(12-34 \mathrm{~mm} \mathrm{Hg})$ & $<0.001$ \\
\hline
\end{tabular}

Abbreviations: IOP, intraocular pressure; $\mathrm{SD}$, standard deviation.

a Denotes statistical significance.

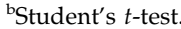

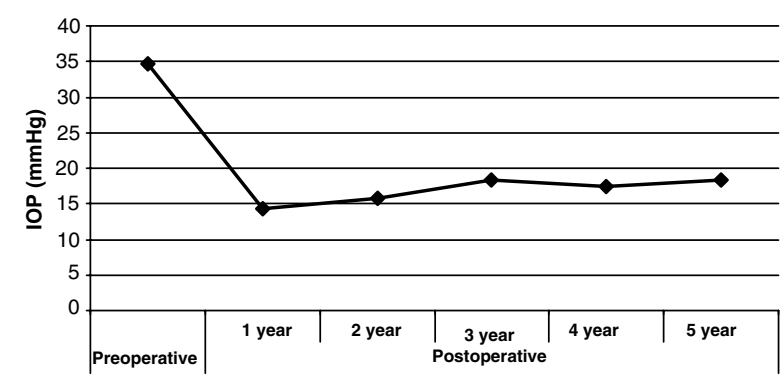

Figure 1 Mean IOP changes from baseline to last follow-up $(P<0.001)$

percentage of reduction in IOP was $-58 \pm 0.14$ (Table 2, Figure 1).

\section{Glaucoma medications}

The mean antiglaucoma medication was $2.30 \pm 0.88$ (range 2-3) preoperatively and $1.60 \pm 2.23$ (range $0-3$ ) postoperatively at the most recent follow-up examination $(P<0.001)$. At the last follow-up, five $(25 \%)$ patients required no antiglaucoma medications (Table 1 ).

\section{Surgical success}

Figure 2 shows the cumulative probability of success (Kaplan-Meier life-table analysis) following trabeculectomy + MMC + inferior sclerotomy, with $85 \%$ success at 1 year, $78.5 \%$ success at 2 years, $76.9 \%$ success at 3 years, $70.6 \%$ success at 4 years and $47 \%$ success at 5 years after surgery.

\section{Visual acuity}

The mean preoperative best-corrected visual acuity (BCVA) was $0.24 \pm 0.15$ (range 0.6-0.05) preoperatively and was $0.18 \pm 0.14$ (range $0.5-0.01$ ) postoperative with Snellen chart $(P=0.144)$. BCVA improved in two $(10 \%)$ 


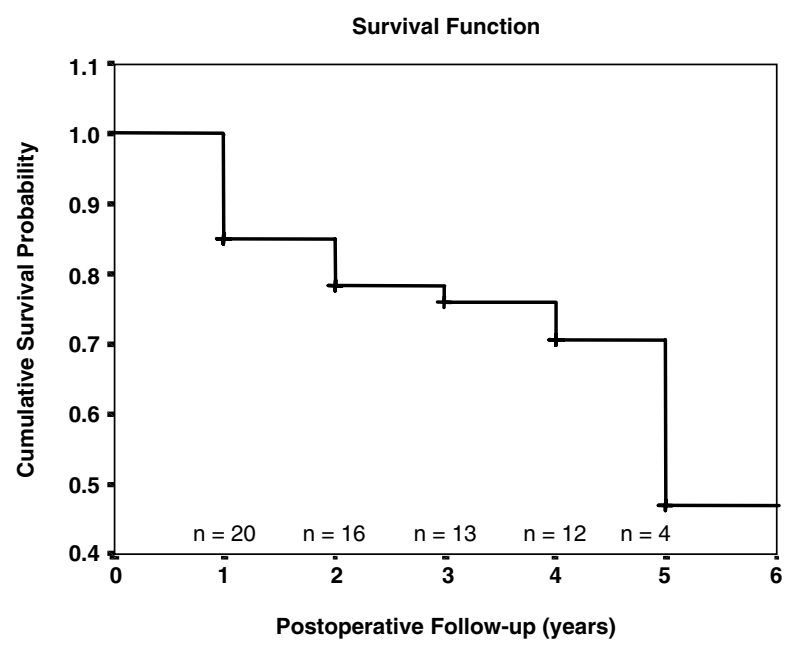

Figure 2 Kaplan-Meier survival curves showing probability of IOP control versus years of follow-up after surgery in patients with nanophthalmos.

Table 3 Postoperative complications

\begin{tabular}{lcc}
\hline & $\begin{array}{c}\text { Complications early } \\
(0-3 \text { months })(\%)\end{array}$ & $\begin{array}{c}\text { Complications late } \\
(>3 \text { months })(\%)\end{array}$ \\
\hline $\begin{array}{l}\text { Choroidal } \\
\text { detachment }\end{array}$ & $10(50 \%)$ & $5(25 \%)$ \\
$\begin{array}{l}\text { Shallow } \\
\text { anterior } \\
\text { chamber }\end{array}$ & $4(20 \%)$ & - \\
$\begin{array}{l}\text { Encapsulated } \\
\text { bleb }\end{array}$ & $3(15 \%)$ & - \\
Cataract & - & $7(35 \%)$ \\
\hline
\end{tabular}

patients or remained within two lines of preoperative visual acuity in five $(25 \%)$ patients in a total of seven $(35 \%)$ patients. Visual acuity decreased in 13 (65\%) patients but no eye lost vision. Sequels of choroidal detachment (five patients, 25\%) and retinal folds (four patients, 20\%) were the most frequent reasons for visual decrease, followed by epiretinal membrane (two patients, $10 \%)$. Two (10\%) patients had a loss of visual acuity to hand motions because of glaucomatous optic nerve damage and severe macular pucker.

\section{Complications}

The most common complication in the early postoperative period ( $0-3$ months) was choroidal detachment in $10(50 \%)$ patients and none of them was treated with laser suture lysis. Of the 10 patients with choroidal detachment, eight $(40 \%)$ were treated conservatively and it resolved within 2 months. Two (10\%) patients had choroidal detachments that were drained. Shallow anterior chamber was seen in four $(20 \%)$ patients. The anterior chamber of the two patients was reformed during the first postoperative week because of lens-corneal touch. Out of the three (15\%) patients with encapsulated bleb, two (10\%) underwent needling + MMC injection.

The most common late postoperative complication (>3 months) that required additional surgery was cataract formation (seven patients, 35\%). Cataract extraction was performed in five (25\%) patients. All of the patients had cataract extraction with posterior chamber intraocular lens implantation, four by phacoemulsification and one by extracapsular cataract extraction.

The second most common late complication was late choroidal effusion (five patients, 25\%). In these eyes, glaucoma surgery was successful for controlling the IOP but uveal effusion occurred after 1-4 years later. In two eyes, choroidal detachment was associated with nonrhegmatogenous retinal detachment. Choroidal effusion was regressed in three (15\%) patients with cycloplegics and massive anti-inflammatory therapy. Inferior sclerotomy and drainage of suprachoroidal fluid were conducted in two (10\%) patients (Table 3)

\section{Discussion}

The simple microphthalmos is essentially a short eye in terms of total axial length (at least 2 SD below the mean for age) with otherwise normal morphological appearance. Nanophthalmos is a form of microphthalmos in which the axial length of a grossly normal globe is $<20.5 \mathrm{~mm}$. The size of lenses in nanophthalmos is within normal ranges, therefore the lens/globe volume ratio, which is $4 \%$ for normals, increases up to the pathological level of $10-30 \%$. The mean axial length was $17.59 \pm 1.65 \mathrm{~mm}$ and the mean lens thickness was $5.00 \pm 89 \mathrm{~mm}$ in our study group being comparable with high lens/globe volume ratio.

Nanophthalmos is often associated with varying degrees of angle-closure glaucoma. A relative pupillary block etiology secondary to posterior 'pushing' mechanism is the most common cause of angle closure glaucoma, which eventually leads to peripheral anterior synechia (PAS) formation. The anterior chamber angle can also be closed by physical displacement of the peripheral iris by anteriorly rotated ciliary processes when nanophthalmos presents with annular ciliochoroidal effusion and ciliary body detachment. ${ }^{6}$

Response to medical treatment is poor and miotics may even make the condition worse by relaxing the lens zonules in these patients. Laser iridotomy is very beneficial in the early stage of glaucoma to eliminate the pupillary block component before the occurrence of PAS 
formation. ALPI is another laser treatment of choice if the anterior chamber remains appositionally closed after iridectomy. ${ }^{7}$

When PAS have developed, intraocular surgery may be required. Surgery is considered as a last resort because it is often followed by a considerable number of complications. Sudden decompression of the globe during surgery may trigger the development of massive uveal effusion, which may lead to secondary retinal detachment, intraocular hemorrhage and malignant glaucoma and loss of vision. Singh et $a l^{3}$ found that $60 \%$ of the 15 patients who had filtration surgery for glaucoma failed to achieve control and $86.6 \%$ suffered visual loss. In 1997, we reported clinical course of glaucoma and early results of trabeculectomy $+\mathrm{MMC}+$ inferior sclerotomy in a small group of patients with nanophthalmos. ${ }^{8,9}$ Mean IOP dropped from 39.3 to $19.3 \mathrm{~mm} \mathrm{Hg}$ and none had postoperative uveal effusion, retinal detachment, visual loss or other major complications in the eyes of two patients in one of our studies. ${ }^{9}$ This study is the continuum of the previous two and it is the first one comparing the long-term results of trabeculectomy $+\mathrm{MMC}+$ inferior sclerotomy for the patients with nanophthalmos. The mean IOP dropped to $21.41 \pm 7.34 \mathrm{~mm} \mathrm{Hg}$ at last visit from $34.6 \pm 0.3 \mathrm{~mm} \mathrm{Hg}$ preoperatively $(P<0.05)$ Kaplan-Meier survival analysis demonstrated the success probabilities of 85, 78.4, 76.9, 70.6 and $47 \%$ at the first, second, third, fourth and fifth year, respectively, in our study.

Despite the good IOP control, choroidal detachment was found in 10 patients $(50 \%)$ in the early period in our study. But most of them were transient and only two $(10 \%)$ of them were drained postoperatively. The association of nanophthalmos with uveal effusion was first reported by Brockhurst. ${ }^{10}$ The uveal effusion may develop either from the thickening of sclera and the reduced scleral permeability to proteins, or from the dense collagen around the vortex veins and the resulting compression of venous drainage channels. Thickening of the sclera has been implicated in the pathogenesis of uveal effusion and serous retinal detachment in patients with nanophthalmos. ${ }^{11-14}$

Brockhurst ${ }^{15}$ introduced vortex vein decompression with lamellar scleral resection, which reduced high venous pressure in choriocapillaris, thereby reducing the leakage of fluid and proteins. However, the surgery was difficult to perform because considerable bleeding occurred during removal of the sclera around the vortex vein. Johnson and Gass ${ }^{16}$ reported that uveal effusion could be treated by lamellar scleral resection and sclerotomy.Calhoun ${ }^{17}$ had reported three cases of nanophthalmos with sclerotomy that had resulted in vitreous hemorrhage, exudative retinal detachment and vitreous bands and all the eyes lost vision. Performing a sclerotomy is another alternative procedure for postoperative drainage. Jin and Anderson ${ }^{4}$ described a V-shaped full-thickness sclerotomy over the pars plana and left it unsutured to provide ongoing drainage postoperatively and they believed that sclera need not to be removed, but that it is sufficient to form opening channels for transscleral drainage and leave it open to provide a persisting route for escape of proteineous suprachoroidal fluid.In our operations, we used inferior full-thickness sclerotomies over the pars plana and left it unsutured to provide continuous drainage

postoperatively. A posterior scleral weakness is at risk of rupture with vitreous and retinal incarceration, but the anterior site is better protected by thicker uveal layer and firm vitreous base. We believe that, it is sufficient to make inferior anterior drainage incisions and leave it open to provide a persistent route for escape of dense suprachoroidal fluid.

The sudden lowering of the IOP to 0 when the globe is opened surgically contributes to the rapid progression of the uveal effusion in patients with nanophthalmos. We modified our classical trabeculectomy technique with preplaced scleral flap sutures, tight suture closure and leaving viscoelastic material in the anterior chamber to overcome intraoperative choroidal effusion. One might argue that early laser suture lysis might aggravate the early postoperative choroidal effusion, but none of our patients who had undergone laser suture lysis developed early postoperative choroidal effusion.

Late choroidal effusion occurred in five $(25 \%)$ patients with two $(10 \%)$ of whom associated with nonrhegmatogenous retinal detachment despite good IOP control. Recurrence of the choroidal detachment after scleral resections was reported, necessitating a second operation with redissection of the sclera. ${ }^{4,18}$ Histological examination has shown that regeneration of both scleral tissue and fibrous scar tissue in specimens excised from previous sclerotomy sites. ${ }^{18}$ Krohn and Seland ${ }^{19}$ described a partial thickness sclerotomy with a central sclerotomy, covered by a thin film of absorbable gelatine to reduce ingrowth of fibrous tissue in the sclerectomized area. Choroidal effusion was regressed with conservative therapy in three $(15 \%)$ patients, but inferior sclerotomy and drainage of suprachoroidal fluid were conducted in two (10\%) patients.

The high refractive errors leading to ametropic amblyopia and macular changes are the leading cause of reduced vision in childhood nanophthalmos. ${ }^{20} \mathrm{In}$ contrast, the leading causes of visual loss in adults with nanophthalmos are glaucoma and the high rate of complications following intraocular surgery., ${ }^{3,4}$ Visual acuity improved or remained within two lines of preoperative levels in seven $(35 \%)$ patients in our study. 
Glaucomatous optic nerve damage contributed to visual acuity decrease only in two (10\%) patients in our study. Sequels of choroidal detachment (five patients, 25\%) were the most frequent reason for visual decrease. Retinal folds were also seen in four (20\%) patients. A disparity in growth between the sclera and retina probably gave rise to the retinal folds as seen in our patients and reported by others. ${ }^{21,22}$

We report the results of a retrospective study of trabeculectomy $+\mathrm{MMC}+$ sclerotomy in patients with nanophthalmos. The results of this study show that IOP control was achieved in high proportion of our patients, but uveal effusion is a major problem and cataract surgery is the most probable option in patients with nanophthalmos.

\section{References}

1 Duke-Elder S. Anomalies in the size of the eye. In Duke Elder S, (ed). System of Ophthalmology, Vol 3 pt 2. CV Mosby: St Louis, MA, 1964, pp 488.

2 Kimbrough RL, Trempe CS, Brockhurst RJ, Simmons RJ. Angle-closure glaucoma in nanophthalmos. Am J Ophthalmol 1979; 88: 572-579.

3 Singh OS, Simmons RJ, Brockhurst RJ, Temple CL. Nanophthalmos: a perspective on identification and therapy. Ophthalmology 1982; 89: 1006-1012.

4 Jin JC, Anderson DR. Laser and unsutured sclerotomy in nanophthalmos. Am J Ophthalmol 1990; 109: 575-580.

5 Auffarth GU, Blum M, Faller U, Tetz MR, Volcker HE. Relative anterior microphthalmos: morphometric analysis and its implications for cataract surgery. Ophthalmology 2000; 107: 1555-1560.

6 Burgoyne C, Tello C, Katz LJ. Nanophthalmia and chronic angle closure glaucoma. J Glaucoma 2002; 11: 525-528.

7 Ritch R, Chang BM, Liebmann JM. Angle closure in younger patients. Ophthalmology 2003; 110: 1880-1889.

8 Altintas AK, Acar MA, Yalvac IS, Kocak I, Nurozler A, Duman S. Autosomal recessive nanophthalmos. Acta Ophthalmol Scand 1997; 75: 325-328.
9 Kocak I, Altintas AK, Yalvac IS, Nurozler A, Kasim R, Duman S. Treatment of glaucoma in young nanophthalmic patients. Int Ophthalmol 1997; 20: 107-111.

10 Brockhurst RJ. Nanophthalmos with uveal effusion: a new clinical entity. Arch Ophthalmol 1975; 93: 1289-1299.

11 Yamani A, Wood I, Sugino I, Wanner M, Zarbin MA. Abnormal collagen fibrils in nanophthalmos: a Clinical and histologic study. Am J Ophthalmol 1999; 127: 106-108.

12 Shiono T, Shoji A, Mutoh T, Tamai M. Abnormal sclerocytes in nanophthalmos. Graefe's Arch Exp Ophthalmol 1992; 230: 348-351.

13 Stewart III D, Streeten BW, Brockhurst RJ, Anderson DR, Hirose T, Gass DM. Abnormal scleral collagen in nanophthalmos. An ultrastructural study. Arch Ophthalmol 1991; 109: 1017-1025.

14 Yue BY, Kurosawa A, Duvall J, Goldsberg MF, Tso MO, Sugar J. Nanophthalmic sclera. Morphologic and tissue culture studies. Ophthalmology 1986; 93: 534-541.

15 Brockhurst RJ. Vortex vein decompression for nanophthalmic uveal effusion. Arch Ophthalmol 1980; 98: 1987-1990.

16 Johnson MW, Gass DM. Surgical management of the idiopathic uveal effusion syndrome. Ophthalmology 1990; 97: 778-785.

17 Calhoun Jr FP. The management of glaucoma in nanophthalmos. Trans Am Ophthalmol Soc 1975; 73: 97-122.

18 Uyama M, Takahashi K, Kozaki J, Tagami N, Takada Y, Ohkuma H. Uveal effusion syndrome: clinical features, surgical treatment, histological examination of the sclera, and pathophysiology. Ophthalmology 2000; 107: 441-449.

19 Krohn J, Seland JH. Exudative retinal detachment in nanophthalmos. Acta Ophthalmol Scand 1998; 76: 499-502.

20 Sener EC, Mocan MC, Sarac OI, Gedik S, Sanac AS. Management of strabismus in nanophthalmic patients. A long-term follow-up. Ophthalmology 2003; 110: 1230-1236.

21 Serrano JC, Hodkings PR, Taylor DSI, Gole GA, Kriss A. The nanophthalmic macula. Br J Ophthalmol 1998; 82. 276-279.

22 Kharillah M, Messaoud R, Zaouali S, Ben Yahia S, Ladjimi A, Jenzri S. Posterior segment changes associated with posterior microphthalmos. Ophthalmology 2002; 109: 569-574. 